\title{
Diffusion and Magnetotransport in Ferrofluids Containing Chain-Shaped Aggregates
}

\author{
A. Yu. Zubarev \\ Ural Federal University, pr. Lenina 51, Yekaterinburg, 620083 Russia \\ Received February 13, 2012
}

\begin{abstract}
The effect of chain structures on particle diffusion in nanodisperse ferrofluids subjected to external magnetic fields is studied. It is shown that the chain formation imparts very strong anisotropy to the diffusion properties of ferrofluids: the diffusion coefficient along a field appears to be approximately two orders of magnitude higher than that in the transverse direction. The presence of the chains changes the particle magnetophoresis coefficient significantly.
\end{abstract}

DOI: $10.1134 / \mathrm{S} 1061933 X 1301016 \mathrm{X}$

\section{INTRODUCTION}

Ferrofluids (magnetic fluids) are colloidal solutions of single-domain ferromagnetic particles in nonmagnetic liquid media. The particle diameter of common magnetic fluids varies in the range of $7-20 \mathrm{~nm}$. In order to avoid irreversible coagulation, the particles are coated with special protecting layers having a thickness of 2-3 nm. Depending on the type of ferrofluid, they are stabilized with either surfactant molecules or electrostatic forces.

For nearly 20 years, ferrofluids have been intensely investigated and widely used owing to their unique physical properties. The diffusion and magnetic kinds of transport of ferroparticles are involved in many fields of technological application of magnetic fluids and determine the distribution of particles in the gravitational and nonuniform magnetic fields. Therefore, the study of the transport phenomena in ferrofluids is of interest from the viewpoint of the development of both the scientific basis for the application of these systems and the general theory of transfer in dispersion media.

In the case of ultimately diluted systems, in which any interparticle interactions may be ignored, the particle diffusion coefficient in ferrofluids is determined by the classical Einstein formula. When the interparticle interactions are significant, the diffusion coefficient depends on the particle concentration and external magnetic field. The coefficients of diffusion and magnetic transport in magnetic fluids were theoretically investigated in [1-4] with allowance for magnetic, hydrodynamic, and steric interparticle interactions. In those works, it was, in particular, shown that the effective diffusion coefficient was anisotropic; i.e., its value in the direction of an applied magnetic field differs from that in the transverse direction.

The cases of moderate and weak interparticle interactions, in which the particles could not aggregate, were considered in the aforementioned works. At the same time, it is well known that, when the energy of the magnetic interparticle interactions in ferrofluids markedly exceeds thermal energy $k T$, the particles can form linear chains or dense bulk droplet-shaped aggregates (see, e.g., [5-9]).

The bulk aggregates have sizes of about $1 \mu \mathrm{m}$; they have repeatedly been observed with ordinary microscopes (see, e.g., [5-7]). Since the sizes of particles in common ferrofluids are smaller than the visible light wavelength, their linear chains cannot be registered by the optical methods. However, such aggregates have been observed in thin layers of ferrofluids by electron microscopy $[8,9]$.

It is known $[10,11]$ that the presence of the aggregates strongly affects the dynamic and, in particular, rheological properties of ferrofluids. It is reasonable to assume that aggregates also affect the transport properties, in particular, diffusion and magnetophoretic phenomena, in these systems.

Note that many modern ferrofluids, especially those composed of magnetite particles, are polydisperse and may have rather wide particle size distributions [10]. The majority of particles in such systems are too small to form any aggregates and structures. Chain- and droplet-shaped aggregates are formed by the largest particles, the concentration of which is, as a rule, low. Therefore, we can hardly expect a substantial effect of these structures on the total mass transfer in magnetic fluids.

However, in recent years, ferrofluids have been synthesized, in which the interaction of the majority of particles is sufficiently strong for aggregation. These systems include, e.g., ferrofluids based on cobalt nanoparticles [11] and ferrofluids containing clustered particles composed of standard ferromagnetic nanoparticles bonded by polymeric shells $[12,13]$. Due to the presence of a large number of single-domain par- 
ticles in a cluster, the clusters strongly interact with one another and unite into aggregates. Experimental data show that these composite ferrofluids combine the sedimentation stability of standard ferrofluids with the unique rheological properties of magnetorheological suspensions.

In ferromagnetic fluids based on cobalt nanoparticles, clusters, etc., that are characterized by rather strong interparticle interactions, the majority or a substantial fraction of particles may occur in an aggregated state. Therefore, the influence of aggregates on their transport properties may be rather strong. Moreover, analysis of magnetodiffusion transport in chaincontaining ferrofluids is of importance in connection with problems concerning the magnetic separation of polydisperse ferrofluids, during which the largest particles capable of forming chain-shaped and other structures are separated from the main volume of a ferrofluid with the help of a nonuniform magnetic field.

The goal of this work was to analyze the effect of chain-shaped structures on diffusion and magnetophoretic mass transfer in ferrofluids containing strongly interacting particles.

\section{PHYSICAL MODEL AND BASIC APPROXIMATIONS}

We use the simplest model developed in [14] for chain-shaped aggregates. Despite its simplicity, use of this model has resulted in the adequate description of the rheological properties of different magnetic fluids formed from both single-domain and clustered particles [15-18].

Within the framework of this model [14-18], particles are considered to be identical to ferromagnetic beads with diameter $d$ and magnetic moment $m$ frozen into the bulk particle. It is assumed that the particles can unite to form chains, which are considered specific heterofluctuations of density. Any interchain interactions are neglected.

As in [14-18], the energy of the directed dipoledipole interaction between adjacent particles in a chain is noticeably higher than $k T$. Note that this is the necessary condition for the formation of aggregates in a ferrofluid. Therefore, the thermal fluctuations of the chain shape and orientation of particle moments relative to the chain axis are ignored. In other words, a chain is considered to be a straight rodlike aggregate in which the moments of particles are directed along its axis. Validity criteria of this approximation have been given in [14]. In that work, it has, in particular, been shown that this approximation is justified when inequality $\varepsilon>\kappa$ is fulfilled $\left(\varepsilon=\frac{\mu_{0}}{2 \pi} \frac{m^{2}}{d^{3} k T}, \kappa=\mu_{0} \frac{m H}{k T}\right.$, where $H$ is the local magnetic field and $\mu_{0}$ is the magnetic permeability of vacuum); i.e., the energy of the interparticle dipole interaction is higher than the energy of particle interaction with the local magnetic field. Finally, we take into account only the magnetic interaction between adjacent particles in a chain.

The representation of the chains as straight rodlike aggregates is, of course, a very strong simplification. A model of flexible chains subjected to an arbitrary magnetic field was developed in [19]. However, it leads to more complex and awkward calculations than the model of straight chains. At the same time, the estimates obtained for ferrofluid magnetization using the model of rodlike chains are adequate at least by order of magnitude, provided that the conditions of its applicability are satisfied [19].

\section{THERMODYNAMIC FUNCTIONS OF MAGNETIC FLUIDS}

Let us consider an elementary representative volume of a ferrofluid. This volume contains very many ferroparticles, and its sizes are assumed to be small as compared with all other linear sizes of the problem, including the characteristic length of variations in the particle concentration. The possibility of the isolation of this volume is the necessary condition for using continual methods for describing transfer processes. Without loss of generality, this volume may be taken to be equal to unity. Let us denote the number of chains composed of $n$ particles and contained in the unit volume of a medium as $g_{n}$. We assume that, at any time moment, the condition of the local thermodynamic equilibrium is fulfilled and each unit elementary volume may be considered to be thermodynamically equilibrium. In terms of the used approximation, free energy $F$ of this volume may be represented in the following form [14]:

$$
F=k T \sum_{n=1}^{\infty}\left[g_{n} \ln \frac{g_{n} V}{\mathrm{e}}-g_{n} \varepsilon(n-1)-g_{n} \ln \frac{\sinh (\kappa n)}{\kappa n}\right],
$$

where $v$ is the particle volume.

The first term in square brackets in Eq. (1) corresponds to the entropy of an ideal gas composed of chains. The second term in the model of straight rodlike chains describes the dimensionless energy of the interaction between adjacent particles, and the third term in the same approximation takes into account the Langevin free energy of the interaction of the chains with local magnetic field $H$.

The equilibrium state of the considered elementary volume is inconsistent with distribution function $g_{n}$ that provides minimum free energy $F$ given satisfied condition of normalization

$$
\sum_{n=1}^{\infty} n g_{n}=\frac{\varphi}{V},
$$

where $\varphi$ is the volume fraction of particles in the given elementary volume. 
The standard calculations (see [14] for details) yield the following expression for the $g_{n}$ function:

$$
g_{n}=\frac{x^{n}}{V} \frac{\operatorname{sh}(\kappa n)}{\kappa n} \exp (-\varepsilon) .
$$

Here, parameter $x$ is the Lagrange undetermined multiplier, which is calculated by substituting relation (3) into condition (2). As a result, we obtain the following:

$$
x=\frac{2 y \cosh (\kappa)+\sinh (\kappa)-\sqrt{(2 y \cosh (\kappa)+\sinh (\kappa))^{2}-4 y^{2}}}{2 y},
$$

where $y=\kappa \varphi \exp (\varepsilon)$.

Substituting expressions (3) and (4) into (1), we find free energy $F$ in the explicit form as a function of local volume concentration $\varphi$ and local field $H$. This expression enables one to determine any thermodynamic parameters characterizing a magnetic fluid. For example, local magnetization $\mathbf{M}$ is

$$
\begin{gathered}
\mathbf{M}=-\frac{\mathbf{H}}{\mu_{0} H} \frac{\partial F}{\partial H}=m \sum_{n=1}^{\infty} n \mathrm{~L}(\kappa n) g_{n} \frac{\mathbf{H}}{H}=\chi \mathbf{H}, \\
\chi=12 \frac{\varepsilon}{\kappa} v \sum_{n=1}^{\infty} n \mathrm{~L}(\kappa n) g_{n} .
\end{gathered}
$$

Here, $\chi$ is the ferrofluid magnetic susceptibility and $\mathrm{L}(x)$ is the Langevin function.

For chemical potential $\mu$ of the particles, we, after simple calculations, obtain the following:

$$
\mu=v \frac{\partial \mathrm{F}}{\partial \varphi}=k T(\ln x-\varepsilon) .
$$

\section{PARTICLE FLUX}

According to the general Batchelor-Einstein formula [20], the flux density $\mathbf{j}_{n}$ of particles contained in $n$-particle chains may be represented as follows:

$$
\mathbf{j}_{n}=-n g_{n} \boldsymbol{\beta}_{n} \cdot \nabla \mu .
$$

Here, $\boldsymbol{\beta}_{n}$ is the hydrodynamic mobility tensor of a chain. The explicit form of its components will be discussed below. The tensor-type character of the chain mobility is associated with the anisotropic shape of the chain and, generally speaking, with an arbitrary orientation of vector $\mathbf{j}_{n}$ and the average direction of the chain axis, which is governed by local field $\mathbf{H}$.

Chemical potential $\mu$ of the particles depends on both particle volume concentration $\varphi$ and absolute value $H$ of the local magnetic field.

Therefore, we may write the following:

$$
\nabla \mu=\frac{\partial \mu}{\partial \varphi} \nabla \varphi+\frac{\partial \mu}{\partial H} \nabla H
$$

Relation (8) should be supplemented with the Maxwell equations for the magnetic field induction and field $\mathbf{H}$ per se. Using the expression $\mathbf{M}=\chi(\varphi, \mathbf{H}) \mathbf{H}$, where $\chi$ is determined by relation (5), these equations may be written in the following form:

$$
\operatorname{div}[\mathbf{H}(1+\chi)]=0, \quad \operatorname{curl} \mathbf{H}=0 .
$$

The former of Eqs. (9) may be rewritten as follows:

$$
(1+\chi) \operatorname{div} \mathbf{H}+\frac{\partial \chi}{\partial \varphi}(\mathbf{H} \cdot \nabla) \varphi+\frac{\partial \chi}{\partial H}(\mathbf{H} \cdot \nabla) H=0 .
$$

Combining Eqs. (7), (8), (9), and (10), we express the final expression for flux $\mathbf{j}_{n}$ via the spatial derivatives of concentration $\varphi$. The total particle flux density is obviously equal to

$$
\mathbf{j}=\sum_{n=1}^{\infty} \mathbf{j}_{n}
$$

Since Eq. (10) is, generally speaking, nonlinear, a simple analytical expression for $\mathbf{j}$ cannot be obtained from relations (7)-(10). Two simple, but typical, cases in which such an expression can be derived are considered below.

\section{Magnetic Field Parallel to Concentration Gradient}

Let us denote the average magnitudes of the field and concentration in some physically small volume as $H_{0}$ and $\varphi_{0}$ and write the following relations:

$$
H=H_{0}+h, \varphi=\varphi_{0}+\varphi^{\prime} .
$$

Without loss of generality, it may be assumed that the strong inequalities $|h| \ll H_{0},\left|\varphi^{\prime}\right| \ll \varphi_{0}$ are fulfilled. Indeed, if $H_{0}$ and $\varphi_{0}$ are considered to be average values in some local region, then, when the condition of the continuity of the concentration field is satisfied, we may always select a range sufficiently small for the aforementioned inequalities to be fulfilled. As relations (8)-(10) are spatially local, i.e., determined for each physical point (each physically small volume), the use of the above inequalities in this volume must not lead to errors.

Let us introduce a Cartesian coordinate system, with the $O x$ axis being directed along the local concentration gradient $\nabla \varphi$. Assume that field $\mathbf{H}_{0}$ is also directed along this axis. As follows from the considerations of symmetry, disturbance $\mathbf{h}$ will also have only a component directed along the $O x$ axis. 

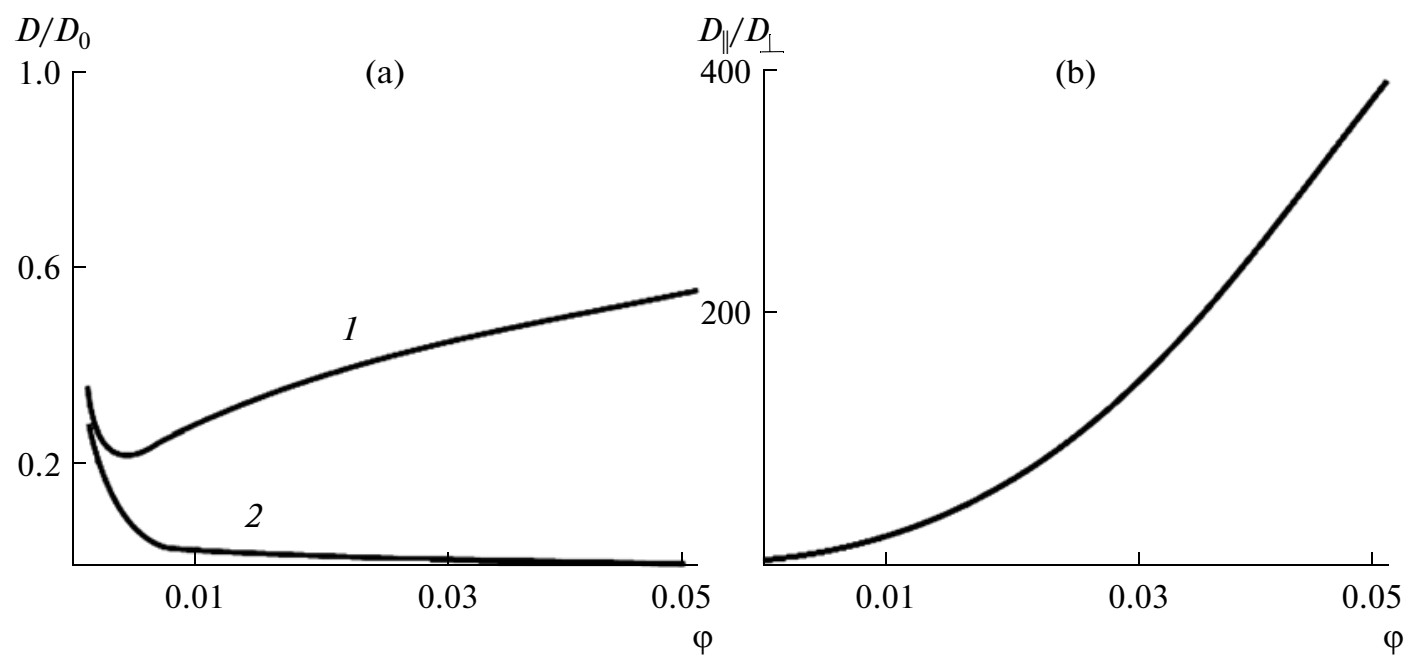

Fig. 1. Dependences of (a) dimensionless coefficients of (1) longitudinal $D_{\|}$and (2) transverse $D_{\perp}$ diffusion and (b) the longitudinal-to-transverse diffusion coefficient ratio on particle volume concentration $\varphi$; calculations have been carried out for $\kappa=2$ and $\varepsilon=6$.

Taking this circumstance into account, after simple transformations of relation (10) in a linear approximations over $h$ and $\varphi^{\prime}$, we obtain

$$
\begin{gathered}
\frac{\partial h}{\partial x}=-B \frac{\partial \varphi^{\prime}}{\partial x}, \\
B(\varphi, H)=H \frac{\partial \chi}{\partial \varphi} \frac{1}{1+\chi+H \frac{\partial \chi}{\partial H}} .
\end{gathered}
$$

Here, the values of $\chi$ and its derivatives are determined for the locally average $H_{0}$ and $\varphi_{0}$ values.

By combining relation (13) with expressions (7), (8), and (11), we derive the following:

$$
\begin{gathered}
j=-\frac{D_{\|}}{V} \frac{\partial \varphi}{\partial x} \\
D_{\|}=\sum_{n=1}^{\infty} \operatorname{vng}_{n} \beta_{n \|}\left(\frac{\partial \mu}{\partial \varphi}-B \frac{\partial \mu}{\partial H}\right) .
\end{gathered}
$$

Here, $\beta_{n \|}$ is the coefficient of the hydrodynamic mobility of a chain for its motion along its axis and $\mathrm{V}$, $D_{\|}$are the particle volume and effective diffusion coefficient at the parallel orientations of the field and concentration gradient. For the aforementioned reasons, there is no difference between $\varphi$ and $\varphi^{\prime}$ in the spatial derivative in relation (14).

\section{Magnetic Field Perpendicular to Concentration Gradient}

After analogous considerations, we obtain $\frac{\partial h}{\partial x}=0$. Relations (7) and (11) yield

$$
\begin{aligned}
j & =-\frac{D_{\perp}}{v} \frac{\partial \varphi}{\partial x}, \\
D_{\perp} & =\sum_{n=1}^{\infty} v n g_{n} \beta_{n \perp} \frac{\partial \mu}{\partial \varphi} .
\end{aligned}
$$

Here, $\beta_{n \perp}$ is the coefficient of the hydrodynamic mobility of a chain for its motion in a direction perpendicular to its axis.

\section{Coefficients of Chain Mobility Tensors $\beta_{n}$}

In the case of an individual particle, coefficient $\beta_{1}$ is determined by the classical Stokes formula for the mobility of a single spherical particle. An analogous coefficient cannot be exactly calculated for a chain because of its complex shape. Here, in order to obtain physically reasonable estimates, we shall simulate a chain by an ellipsoid of revolution with major and minor axes $n d$ and $d$, respectively. Note that the same model was used in [14-18] to determine the rheological characteristics of a ferrofluid.

Mobility coefficients of ellipsoids of revolution may be found in, e.g., [21]. For longitudinal and perpendicular motion relative to the ellipsoid axis, the mobility coefficients are, respectively, equal to

$$
\beta_{n \|}=\frac{1}{16 \pi \eta}\left(\gamma+\alpha_{1} n^{2} a^{2}\right), \quad \beta_{n \perp}=\frac{1}{16 \pi \eta}\left(\gamma+\alpha_{2} a^{2}\right),
$$

where

$$
\alpha_{1}=\int_{0}^{\infty} \frac{d \lambda}{\left(n^{2} a^{2}+\lambda\right) \Delta(\lambda)}, \quad \alpha_{2}=\int_{0}^{\infty} \frac{d \lambda}{\left(a^{2}+\lambda\right) \Delta(\lambda)},
$$

$$
\begin{array}{llll}
\text { COLLOID JOURNAL } & \text { Vol. } 75 & \text { No. } 1 & 2013
\end{array}
$$



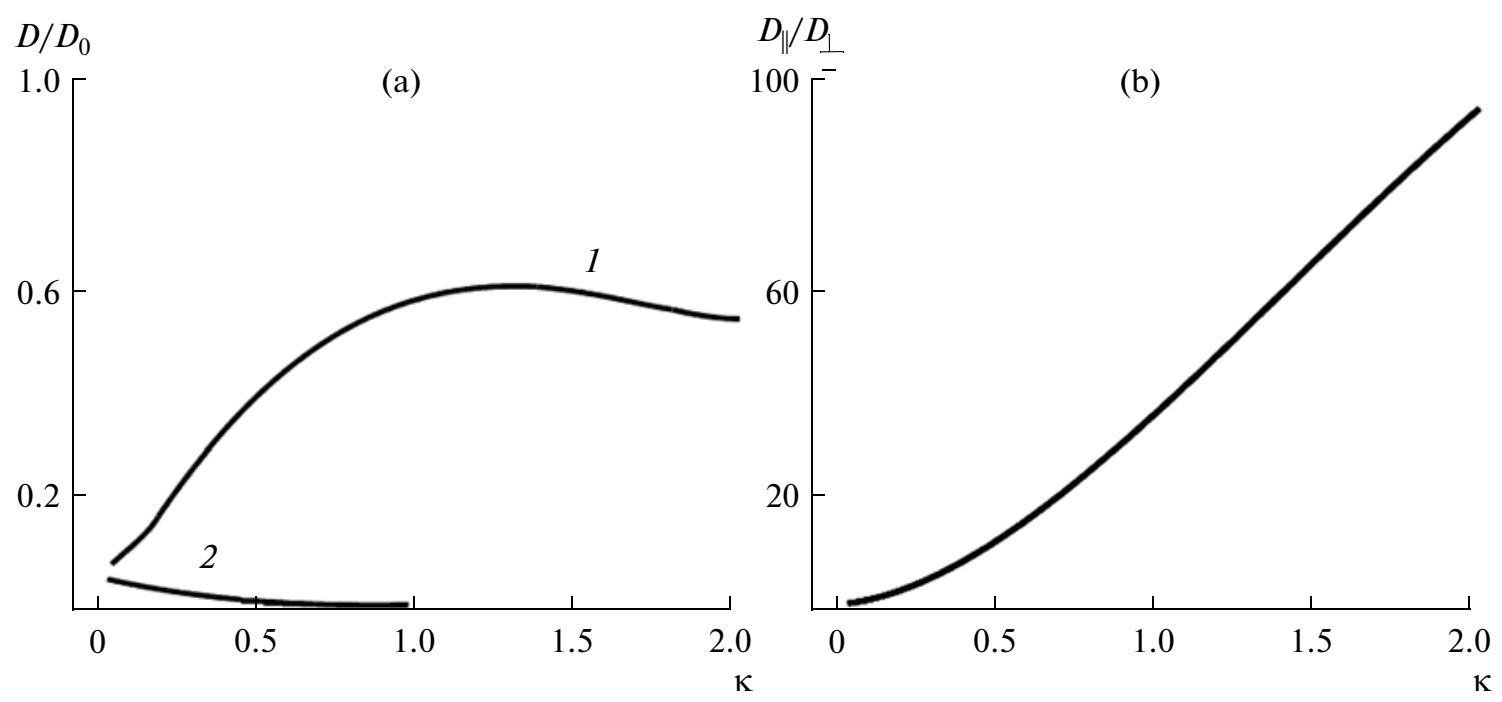

Fig. 2. Dependences of (a) effective diffusion coefficients and (b) the longitudinal-to-transverse diffusion coefficient ratio on dimensionless magnetic field magnitude $\kappa$. The denotations of the curves are the same as in Fig. 1; the calculations have been performed for $\varphi=0.025$ and $\varepsilon=6$.

$$
\gamma=\int_{0}^{\infty} \frac{d \lambda}{\Delta(\lambda)}, \quad \Delta(\lambda)=\sqrt{\left(n^{2} a^{2}+\lambda\right)}\left(a^{2}+\lambda\right) .
$$

Here, $a=d / 2$ is the hydrodynamic particle radius. Coefficients $D_{\|}$and $D_{\perp}$ calculated as functions of concentration $\varphi$ are listed in Fig. 1.

The dependence of $D_{\perp}$ on concentration $\varphi$ is explained by a combination of two similarly directed factors. First, magnetic attraction of particles and their aggregation into chains reduce chemical potential $\mu$ and derivative $\frac{\partial \mu}{\partial \varphi}$ as compared with the individual particles. Second, the chain formation diminishes the effective hydrodynamic mobility of the particles. Both these factors result in decreasing $D_{\perp}-\varphi$ dependence. Along with these factors, the $D_{\|}-\varphi$ dependence is affected by the retraction of the magnetic particles into the region where magnetic field $H$ is higher, i.e., into the region of lower concentrations $\varphi$. This factor is obvious to increase the effective diffusion of the ferroparticles. The combination of the oppositely directed factors results in the nonmonotonic $D_{\|}-\varphi$ dependence.

The dependences of both diffusion coefficients on dimensionless local magnetic field are illustrated in Fig. 2.

A monotonic reduction in $D_{\perp}$ with magnetic field is explained by an efficient increase in the attraction of the magnetic particles with a rise in the field due to an increase in the ordering of their orientations. As a result, chemical potential $\mu$ of the particles decreases as the field magnitude increases. Therewith, the char- acteristic length of the chains enlarges to reduce the effective hydrodynamic mobility of the particles. Dependence of $D_{\|}$on the field is, along with these factors, affected by the retraction of the particles into the region of higher fields, i.e., lower concentrations. This factor of increasing $D_{\|}$obviously becomes stronger with enhancement of the field. The combination of the opposite factors results in nonmonotonic dependence of $D_{\|}$on the field magnitude.

As can be seen from Figs. 1 and 2, the effective diffusion is strongly anisotropic; i.e., in the considered ranges of variations in the particle concentration and magnetic field, the $D_{\|}$and $D_{\perp}$ coefficients differ by nearly one to two orders of magnitude. This great difference is due to, first, the different hydrodynamic mobilities of the chains in the directions along and perpendicular to their axes and, second (in the case of diffusion along the field), the retraction of the particles into regions with lower concentrations, which is caused by the fact that the local magnetic field in them is higher than that in the regions of higher concentrations. This effect is much stronger for chain-containing ferrofluids than for fluids containing individual particles, because the orientations of particle magnetic moments in a chain are closely correlated; therefore, the particle moments are more strongly oriented along the field. As a consequence, the chain-field interaction energy is higher than the total interaction energy of individual particles.

\section{MAGNETOPHORESIS}

Now, let us consider a model case in which concentration $\varphi$ of particles is uniform and their transport is induced by gradient-type field $H$. Maxwell equations 


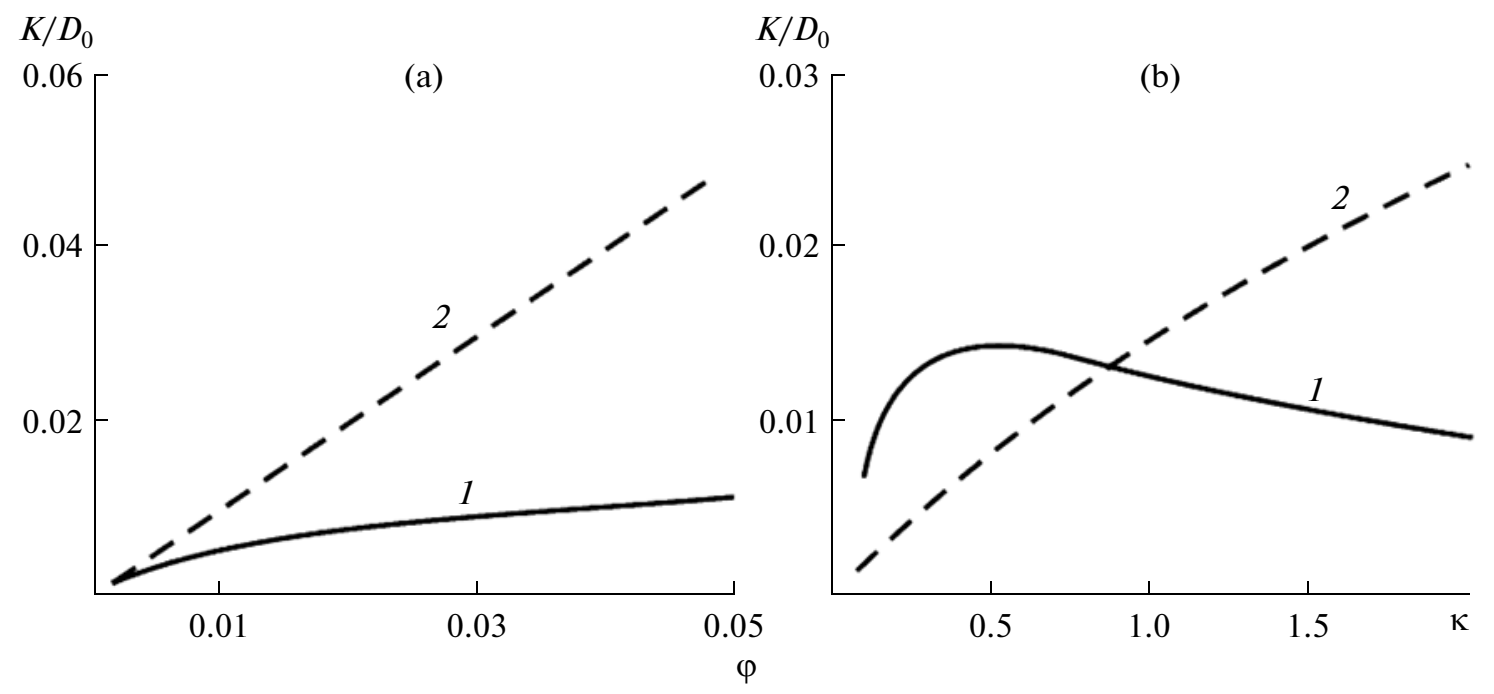

Fig. 3. Dependences of the dimensionless coefficient of magnetophoresis on (a) particle volume concentration at $\kappa=2$ and (b) the dimensionless magnetic field magnitude at $\varphi=0.025$. The calculations have been carried out at $\varepsilon=6$ by formulas ( 1 ) (16) and (2) (17).

(9) and (10) show that, in this case, the magnetic field must vary in the direction perpendicular to the direction of the field. Therefore, transport of the chains will take place in the direction perpendicular to the prevailing orientation of their axes. By combining relations (7), (8), and (11), we derive

$$
\begin{gathered}
\mathbf{j}=K \nabla \kappa, \\
K=-\sum_{n=1}^{\infty} n g_{n} \beta_{n \perp} \frac{\partial \mu}{\partial \kappa} .
\end{gathered}
$$

Here, $K$ may be considered to be the coefficient of particle magnetophoresis. Since chemical potential $\mu$ decreases with an increase in dimensionless magnetic field $\kappa$, coefficient $K$ is positive.

Under the approximation of individual noninteracting particles, it must be taken that, in relation (1), $g_{1}=\varphi / v$ and $g_{n}=0$ when $n \neq 1$. In this case, we have the following:

$$
K_{0}=\frac{\varphi_{V}}{\beta_{0} L(\kappa),} \quad \beta_{0}=3 \pi \eta d .
$$

Here, subscript 0 refers to the coefficients of magnetophoresis and hydrodynamic mobility of individual particles and $\eta$ is the viscosity of a carrier medium.

The calculated $K$ and $K_{0}$ values are presented in Fig. 3. At relatively low magnitudes $\kappa$ of the dimensionless field, the chain formation enhances the effective magnetophoresis coefficient due to the prevailing influence of increasing magnetization of the medium as a result of the correlation of the particle moments in the chains. However, as the magnetic field becomes stronger, the characteristic length of the chains increases to decrease their effective hydrodynamic mobility. As a result, at rather high $\kappa$ values, the hydro- dynamic factor prevails; therefore, coefficient $K$ calculated through formula (16) becomes lower than $K_{0}$ calculated in the approximation of individual particles (17).

\section{CONCLUSIONS}

The effect of chain structures on the diffusion and magnetic transport of particles in nanodisperse ferrofluids has been analyzed in the simplest approximation of noninteracting straight rodlike chains. Calculations have shown that, as a result of the chain formation, a very strong anisotropy develops in the diffusion properties of ferrofluids; i.e., the effective diffusion coefficient in the direction of the field becomes one to two orders of magnitude higher than that in the transverse direction. This is explained by the following phenomena: first, retraction of particles into ferrofluid regions with increased magnitudes of the local magnetic field, i.e., regions with decreased concentrations, and, second, a higher mobility of chains in the directions along their axes than in the transverse direction. The calculations predict a monotonic reduction in the coefficient of the transverse (directed perpendicularly to the local magnetic field) diffusion with both the particle concentration and the magnitude of the local field. The longitudinal (directed along the field) diffusion coefficient nonmonotonically depends on the particle volume concentration and magnetic field.

At low magnitudes of the dimensionless field, the presence of chains enhances the effective coefficient of magnetophoresis as compared with individual noninteracting particles, while, at high magnitudes of the field, it decreases this coefficient.

In spite of the fact that the approximation of noninteracting rodlike aggregates is a strong simplification 
of the system, it, previously, resulted in describing the rheological properties of different magnetic fluids and suspensions. This circumstance leads us to believe that this approximation, at least in principle, adequately describes the effect of the chains on the transport properties of such systems. Indeed, analysis [19] has indicated that the approximation of rodlike chains makes it possible to estimate the chain magnetic moment, at least to an order of magnitude. As was shown in [22], although magnetic and steric interactions of the chains affect their size distribution function, at low particle concentrations, when the conditions for their phase condensation are absent, this effect is not too strong. Therefore, as a first approximation, it may be ignored. As to the hydrodynamic interchain interaction, successful application of the approximation of noninteracting chains for describing the rheological properties of magnetic fluids suggests that this interaction also does not play a key role in the development of their dynamic properties.

It should be noted that, at rather high, but real, values of particle concentration and interparticle magnetic interaction energy, phase condensation of particles into dense bulk droplets takes place in magnetic fluids. Analysis of the transport phenomena under such conditions requires the solution of a separate problem.

\section{ACKNOWLEDGMENTS}

This work was supported by the Russian Foundation for Basic Research, projects nos. 10-01-96002Ural, 10-02-96001-Ural, and 10-02-00034.

\section{REFERENCES}

1. Buevich, Yu.A., Zubarev, A.Yu., and Ivanov, A.O., Magnetohydrodynamics, 1989, vol. 2, p. 39.

2. Morozov, K.I., J. Magn. Magn. Mater., 1993, vol. 122, p. 98.

3. Morozov, K.I., Phys. Rev. E: Stat. Phys., Plasmas, Fluids, Relat. Interdiscip. Top., 1996, vol. 3, p. 3841.

4. Pshenichnikov, A.F., Elfimova, E.A., and Ivanov, A.O., J. Chem. Phys., 2011, vol. 134, p. 184508.

5. Hayers, C.F., J. Colloid Interface Sci., 1975, vol. 52, p. 239.
6. Bacri, J.C. and Salin, D., J. Magn. Magn. Mater., 1983, vol. 9 , p. 48.

7. Islam, M.F., Lin, K.H., Lacoste, D., Lubenski, T.C., and Yodh, A.G., Phys. Rev. E: Stat. Phys., Plasmas, Fluids, Relat. Interdiscip. Top., 2003, vol. 67, p. 021402.

8. Klokkenburg, M., Dullens, R.P.A., Kegel, W.K., Erne, B.H., and Philipse, A.P., Phys. Rev. Lett., 2006, vol. 96, p. 037203.

9. Klokkenburg, M., Erne, B.H., Meedldijk, J.D., Wiedenmann, A., Petukhov, A.V., Dullens, R.P.A., and Phylipse, A.P., Phys. Rev. Lett., 2006, vol. 97, p. 185702.

10. Odenbach, S., Magnetoviscous Effects in Ferrofluids, Berlin: Springer, 2002.

11. Ilg, P. and Odenbach, S., in Colloidal Magnetic Fluids, Odenbach, S., Ed., Berlin: Springer, 2009, p. 249.

12. Dutz, S., Andrea, W., Hergt, R., Muller, R., Oestreich, Ch., Schmidt, Ch., Eopfer, J.T., Zeisberger, M., and Bellemann, M., J. Magn. Magn. Mater., 2007, vol. 311, p. 51.

13. Borin, D. and Odenbach, S., Abstracts of Papers, 12 Int. Conf. on Electrorheological Fluids and Magnetorheological Suspensions, Singapore: World Scientific, 2011, p. 12.

14. Zubarev, A.Yu. and Iskakova, L.Yu., Zh. Eksp. Teor. Fiz., 1995, vol. 107, p. 1534.

15. Zubarev, A.Yu., Fleisher, J., and Odenbach, S., Physica $A$ (Amsterdam), 2005, vol. 358, p. 47.

16. Chirikov, D.N., Fedotov, S.P., Iskakova, L.Yu., and Zubarev, A.Yu., Phys. Rev. E: Stat. Phys., Plasmas, Fluids, Relat. Interdiscip. Top., 2010, vol. 82, p. 051405.

17. Borin, D., Zubarev, A., Chirikov, D., Muller, R., and Odenbach, S., J. Magn. Magn. Mater., 2011, vol. 323, p. 1273.

18. Rodriguez-Arco, L., Lopez-Lopez, M.T., Duran, J.D.G., Zubarev, A., and Chirikov, D., J. Phys.: Condens. Matter, 2011, vol. 23, p. 455101.

19. Mendelev, V.S. and Ivanov, A.O., Phys. Rev. E: Stat. Phys., Plasmas, Fluids, Relat. Interdiscip. Top., 2004, vol. 70, p. 051502.

20. Batchelor, G.K., J. Fluid Mech., 1976, vol. 74, p. 1.

21. Van de Ven, T.G.M., Colloidal Hydrodynamics, New York: Academic, 1989.

22. Iskakova, L.Yu. and Zubarev, A.Yu., Phys. Rev. E: Stat. Phys., Plasmas, Fluids, Relat. Interdiscip. Top., 2002, vol. 66, p. 041405. 